\title{
Simulation of Charge and Mass Distributions of Indium Droplets Created by Field Emission
}

\author{
Anton VanderWyst* \\ Department of Aerospace Engineering, University of Michigan, Ann Arbor, MI, 48109 \\ Andrew Christlieb ${ }^{\dagger}$ \\ Department of Mathematics, University of Michigan, Ann Arbor, MI, 48109 \\ Mark Sussman \\ Department of Mathematics, Florida State University, Tallahassee, FL 32306-4510 \\ Iain D. Boyd ${ }^{\S}$ \\ Department of Aerospace Engineering, Univeristy of Michigan, Ann Arbor, MI 48109
}

Field emission electric propulsion (FEEP) thrusters are useful as $\mu \mathrm{N}, \mu$-radian attitude control devices for satellites. In this study, we simulate in axisymmetric 2D coordinates the emission of charged indium leaving the surface and forming unique droplets. The boundary element method is used to rapidly and accurately calculate the electric field on the fluid surface, which is then advected forward in time using the combined level set and volume of fluid (CLSVOF) method. The probability density function (PDF) of droplet charge to mass ratio is simulated. Each drop influences the size, charge and path of the following new drop.

\section{Nomenclature}

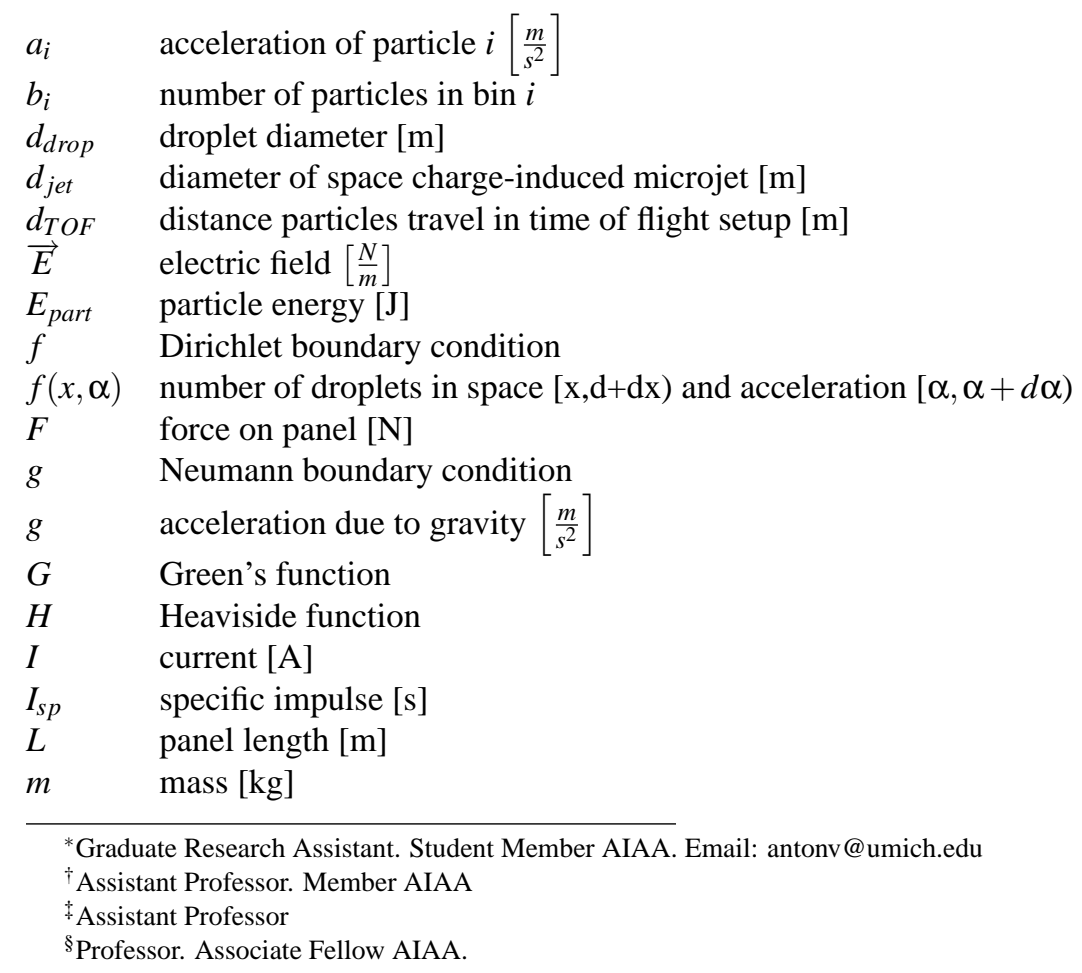




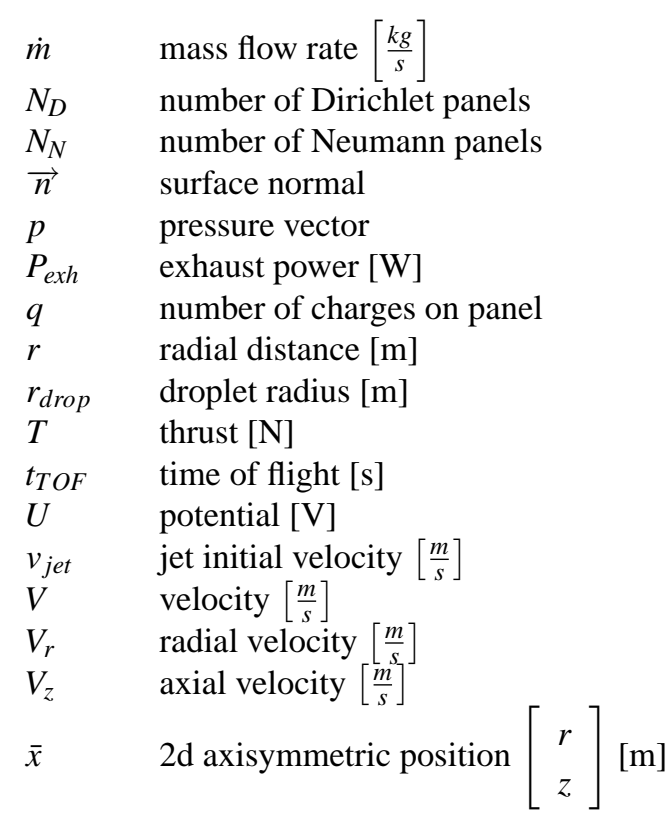

Greek symbols

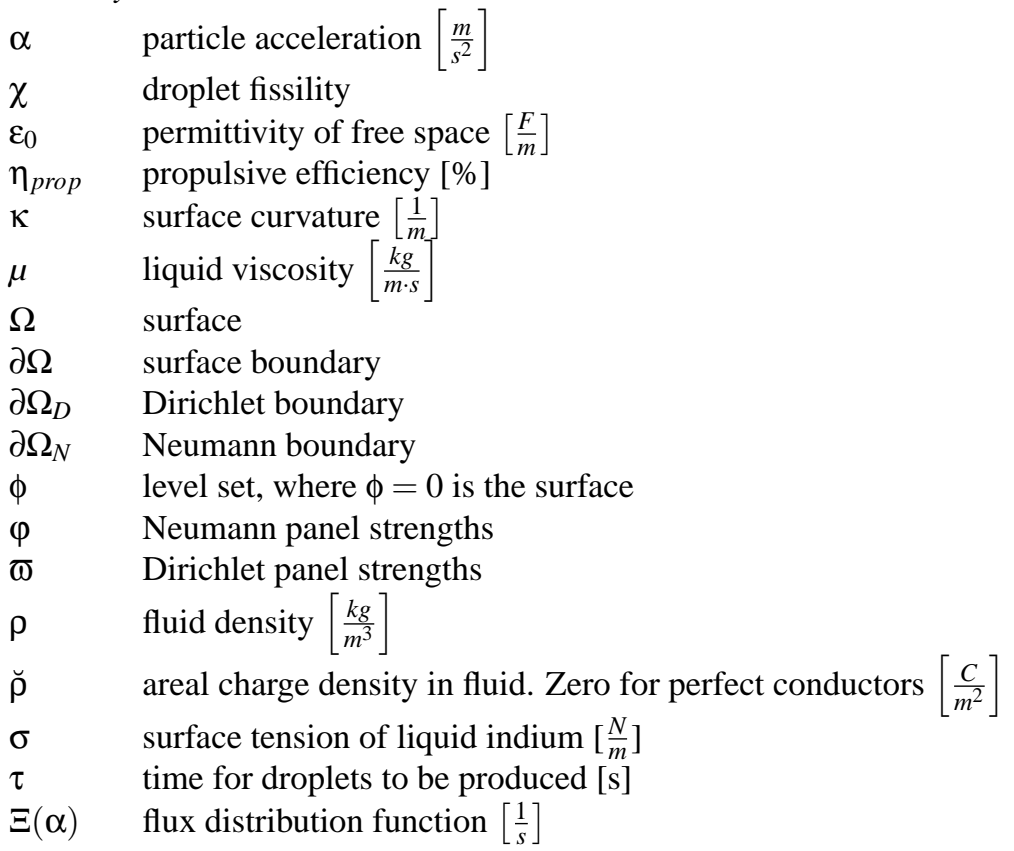

Abbreviations

ARCS Austrian Research Centre - Seiborsdorf

FEEP field emission electric propulsion

$M T C R$ mass to charge ratio $\left[\frac{\text { numberofatoms }}{e}\right]$

TOF time of flight [s]

\section{Introduction}

GIELD emission refers to the process of using a strong electric field to produce a spray of charged ions or droplets. This occurs when two electrodes form a potential difference that pulls on a conducting fluid, but is resisted by 
surface tension and viscosity of the fluid. As a result of this pull, the liquid surface deforms to an equilibrium shape: a cone. The electric field acting at the tip of the cone then causes a jet of ions or droplets to form [1].

The application of field emission to fluids has been studied as early as 1923 by Schottky [2]. Research in this area has established that the necessary potential difference for droplet formation varies by material composition [3, 4]. Field-ion emission with liquid metal was examined initially by Tsong and Müller in the 1960's [5]. Applications of this technology have proven to be numerous, including realms such as: electron microscopy [6], data displays [7], carbon nanotube fluorescence [8], ink jets [9] and thermoelectric coolers [10]. However, a significant possibility for the use of the field emission process to produce thrust in space had been overlooked. When used for propulsion, field emission produces thrust through expelling propellant at high velocity.

One example of a space thruster that combines field emission and electric propulsion is called a field emission electric propulsion thruster, or FEEP. Figure (1) shows the useful range of specific impulse and power for various electric propulsion technologies. Compared to these other designs, FEEP thrusters provide a source of high specific impulse $\left(I_{s p}\right)$, low thrust/power, ultra-low impulse bit electrostatic space propulsion. They have been examined since the 1970's [11, 12] and the ion/droplet plume ratio based on mass flow has been predicted [13, 14]. Several scaling laws about current and voltage, droplet size and specific impulse have been published $[15,16]$.

A space-tested FEEP has been under development in Austria for over a decade [17]. Such thrusters are appropriate for scientific drag-free missions such as LISA [18], Darwin [19], GOCE [20] and SMART-2 [21]. The thruster as built and tested by the Austrian Research Center - Seiborsdorf (ARCS) consists of a tungsten needle covered in liquid indium reacting to an applied electric potential difference between an extractor ring and the needle of $-6 \mathrm{kV}$ $[22,17,16]$. When the field strength at the tip reaches $1 \times 10^{9} \frac{\mathrm{V}}{\mathrm{m}}$, the indium is accelerated over the needle that is about $1 \mathrm{~cm}$ long and $50 \mu \mathrm{m}$ wide and ionized from the surface. Depending on the mass flow rates, either ions or droplets are observed coming from the needle tip. Planar and isometric schematics of a FEEP [23] are shown in Fig. (2).

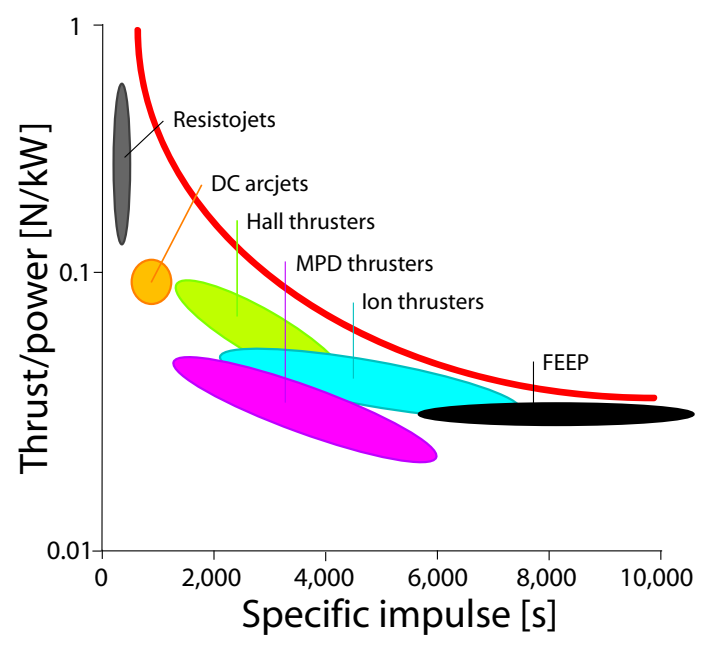

Figure 1. Electric propulsion technology types

ARCS has shown that droplet-driven FEEPs offer maximum thrust. These droplets are important because, compared to ions, while their presence increases thrust it reduces mass efficiency. This trend can be seen from the fact that the exhaust power divided by the thrust is proportional to the specific impulse.

$$
P_{e x h} / T=\frac{1}{2} v=\frac{1}{2} g I_{s p}
$$

As the engine becomes increasingly mass efficient, more power per unit thrust is required. Using Eq. (1) with a fixed maximum power supply, Eq. (2) relates mass flow rate and $I_{s p}$.

$$
\text { const }=T \times I_{s p} \sim \dot{m} I_{s p}^{2}
$$

Therefore higher charged, faster-moving ions produce less thrust with a lower mass flow rate for a given power level than the corresponding droplet emission. In addition, depending on the radial speed of the droplets, their electrostatic 


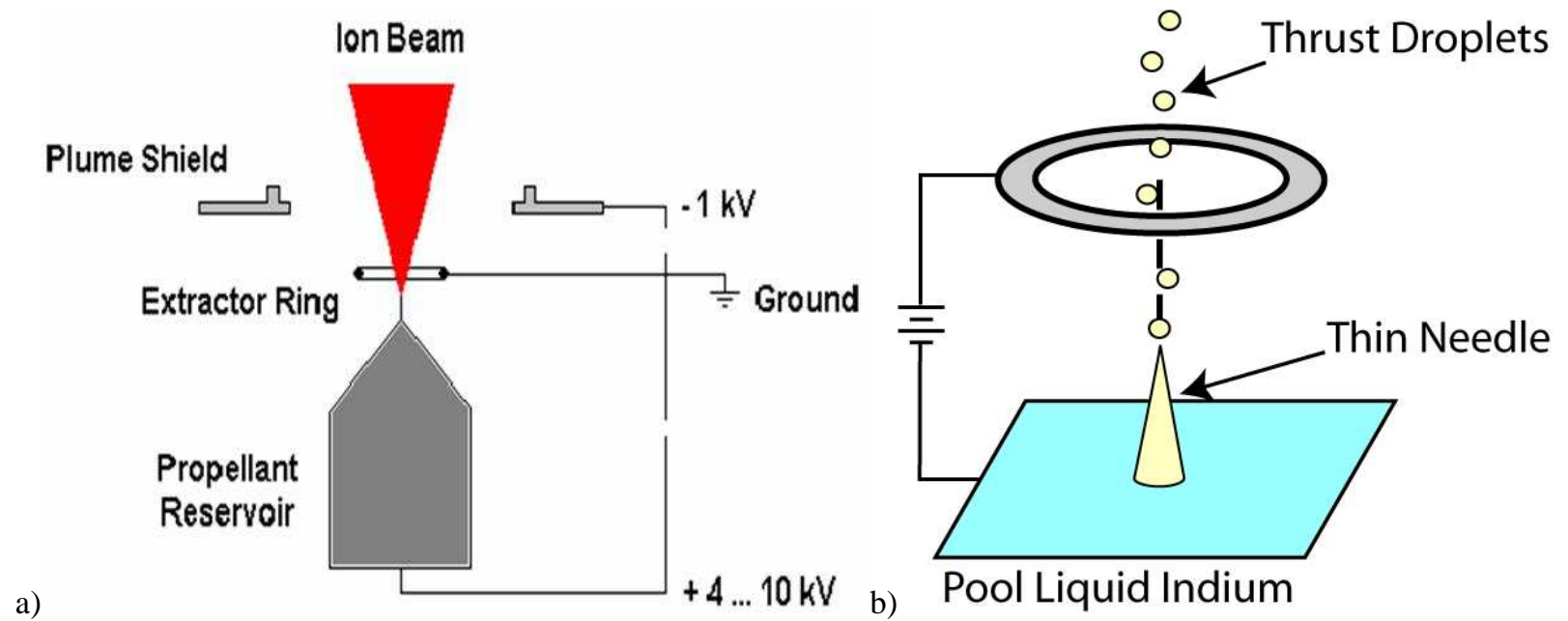

Figure 2. Needle FEEP emitting a) ions and b) droplets

potential at birth and the degree of interception by other droplets, the separation between particles changes. This variation can cause the plume width to vary and impart a net off-axis force to the thruster. In order to delay the onset of a droplet-dominated plume and extend the high specific impulse regime, it is necessary to understand the environment within which these droplets form. So far, no such computational models exist that predict the droplet characteristics of liquid metal field emitters at high currents. Our objective is to create a model that describes droplet behavior for an indium-fed needle FEEP. This paper will discuss the physical governing equations, the level set and boundary element method computational algorithms used to simulate detaching droplets and then present properties for these droplets.

\section{Description of Model}

The simulation model considers an incompressible, isothermal and viscous liquid. The propellant indium is treated as a perfect conductor and its atoms are accelerated by a ring electrode, thereby producing thrust. The two dimensional axisymmetric governing equations for the system are listed as Eqs. (3-4). The electric field $\vec{E}$ is a surface normal force, since all electrons in a conductive material remain on the surface. Conservation of mass and momentum are enforced.

$$
\begin{aligned}
\frac{1}{r} \frac{\partial}{\partial r}\left(r v_{r}\right)+\frac{\partial v_{z}}{\partial z} & =0 \\
(V \cdot \nabla) \mathbf{A} & =\mathbf{B}+\sigma \kappa+q E_{n}
\end{aligned}
$$

The main momentum equation variables are listed in Eq. (4).

$$
\mathbf{A}=\left[\begin{array}{c}
v_{r} \\
v_{z}
\end{array}\right], \mathbf{B}=\left[\begin{array}{c}
-\frac{1}{\rho} \frac{\partial p}{\partial r}+\frac{\mu}{\rho}\left(\nabla^{2} v_{r}-\frac{v_{r}}{r^{2}}\right) \\
-\frac{1}{\rho} \frac{\partial p}{\partial z}+\frac{\mu}{\rho} \nabla^{2} v_{z}
\end{array}\right]
$$

The subfunction variables $v_{r}$ and $v_{z}$ are local velocity vectors along the $r$ and $z$ axes, respectively. The normal electric field is computed by $E_{n}=-\nabla U(\vec{x})$, where $U(\vec{x})$ is the electrostatic potential at position $\vec{x}$. A method of rapidly computing the electrostatic force $q E_{n}$ is described in Sec. (C). Interface curvature (א) [24] is a function only of the surface shape.

\section{Parameter submodels}

The surface tension $(\sigma)$ of liquid indium does not vary rapidly with temperature [25], as indicated by Eq. (5).

$$
\sigma_{\text {In }}\left[\frac{N}{m}\right]=\frac{555-0.12(T[K]-430)}{1000}
$$


The ability of indium to flow over a solid surface is determined largely by its viscosity $(\mu)$ which varies with temperature through the weak exponential relationship shown in Eq. (6). Over the narrow operational range of $440-450 \mathrm{~K}$ when indium is far enough above its melting point to be fully liquid [26], the fluid viscosity changes only 4\% [25].

$$
\mu_{\text {In }}\left[\frac{\mathrm{kg}}{\mathrm{m} \cdot \mathrm{s}}\right]=3 \times 10^{-4} e^{800 / T[K]}
$$

The density of indium $(\rho)$ changes about $0.01 \%$ per degree change [27].

$$
\rho_{\text {In }}\left[\frac{k g}{m^{3}}\right]=1000 \times\left(7.1295-6.7987 \times 10^{-4}(T-273.15)\right)
$$

The assumption of a perfect conductor is very reasonable. As a metal, the conductivity of indium is so high that an electron can travel through the liquid surface along the needle body in 6 picoseconds while the fluid flows at a maximum speed 10 orders of magnitude slower, only $100 \frac{\mu m}{s}$. The assumption of isothermal detached droplets is accurate because only radiation is available to cool them after snap off. For example, detachment occurs in $0.1 \mu \mathrm{s}$, but a $100 \mu \mathrm{m}$ diameter droplet at $450 \mathrm{~K}$ cools less than $0.1 \mathrm{mK}$ in that time [28].

\section{Numerical Implementation}

To simulate the characteristics of FEEP droplets using the model framework previously mentioned, numerical methodologies and algorithms must be chosen. In the following subsections are: descriptions of methods to model material interfaces and rapid determinations of electric fields; theoretical comparison of modeled results to experimental data; and an overview of droplet critical fissility.

For the computational tracking of a surface between solid-liquid and liquid-vacuum interfaces, the determination of the normal electric field $\vec{E}_{n}$ commonly is quite drawn out and error-prone. One successful Eulerian approach discretized the problem using a symmetric, second-order accurate method with evenly spaced mesh points in an irregular domain [29]. Our usage of level sets and the boundary element method improves on that work by providing similar spatial resolution in much less time, handles a changing irregular shape and includes the effects of electrostatic forces on the behavior of a droplet after pinch off.

\section{A. Level sets}

Level sets allow for computational tracking of a surface between discrete interfaces. They were introduced by Osher and Sethian in the late 1980's [30] and have been heavily analyzed over the next couple of decades [31, 32, 33, 34, 35]. Many physical phenomena have been simulated using level sets, including ink jets [36], electrical tomography [37], turbulent channel flow [38], brain scans [39] and radar image processing [40, 41].

Interface tracking using level set computation relies on the determination and movement of the boundary. In FEEP operation, liquid propellant exists between a hard vacuum and a solid tungsten needle. The dominating forces are surface tension, viscosity and electrostatic potential. Here, we present results achieved on irregular domains and shapes. In the simulation, the position of the free surface is updated via the level set equation:

$$
\phi_{t}+V \cdot \nabla \phi=0
$$

where $\phi$ is the level set function; positive in the liquid and negative in the vacuum. $V$ is the interface velocity and is taken to be the fluid velocity inside the fluid and an extension of the fluid velocity for $\phi<0$. Equation (7) states that $\phi$ remains constant on particle paths. If a point starts on the surface $\partial \Omega$ where $\phi=0$, that particle moves through time, but remains on the surface. Figure (3a) shows the $\phi \geq 0$ cross-section of a double-humped shape intersected at 3 different heights while Fig. (3b) demonstrates how the $\phi$ level set values around the rightmost hump reproduce the surface contour [42].

To maintain second order spatial accuracy, a combined level set (CLS) and volume of fluid (VOF) approach is used (CLSVOF). Used in the volume of fluid piecewise linear reconstruction step, the surface normals are computed from the smoothed level set function. A level set reinitialization replaces the existing $\phi$ with the level set function that gives the exact signed normal distance from the surface. The numerical schemes used in the CLSVOF approach are [43]:

1. Velocity and pressure fields are computed based on variable density projection methods [44]. 

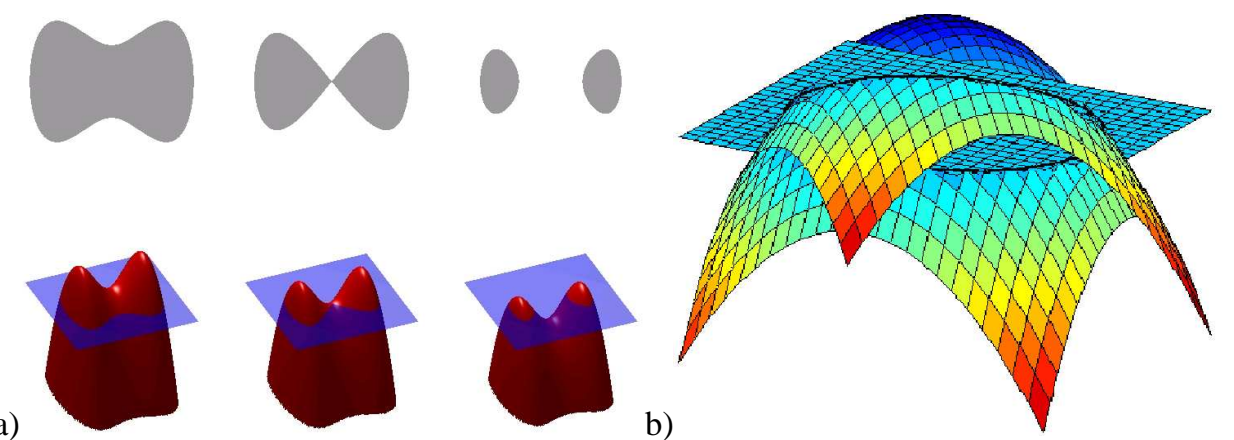

a)
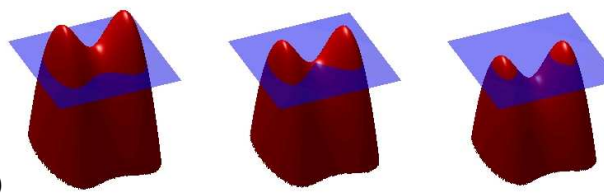

b)

Figure 3. Level set a) surface determination at various heights and b) $\phi$ values around the upper right hump at the highest point.

2. The discretization uses a Godunov second-order upwind predictor-corrector step [45].

3. Time stepping occurs via the Runge-Kutta method for advection and the Crank-Nicholson method for viscous terms [46].

4. The interface curvature is estimated using volume-of-fluid measures.

5. The surfaces are computed using second-order split advection algorithms.

An additional procedure to retain second order spatial accuracy is for the governing Eq. (7) to be solved with a second-order hydrodynamic scheme [47,34]. The density and viscosity are spread out over multiple grid cells in order to avoid sharp changes in pressure and diffusion terms via the smoothed $\varepsilon$-offset Heaviside function of Eq. (8).

$$
H(\phi)\left\{\begin{array}{cc}
1 & \phi>\varepsilon \\
\frac{1}{2}\left(1+\frac{\phi}{\varepsilon}+\frac{1}{\pi} \sin \frac{\pi \phi}{\varepsilon}\right) & |\phi| \leq \varepsilon \\
0 & \phi<-\varepsilon
\end{array}\right.
$$

\section{B. Boundary element method}

A complementary surface simulation technique to level sets is the boundary element method. Commonly, it allows analysis of the behavior of mechanical systems and engineering structures subjected to external loading through heat, mass or inhomogeneous boundary conditions $[48,49]$. These approaches mimic the more common finite element method, except that boundary elements span only the boundaries of the problem instead of the entire volume. This restriction allows for faster computation due to fewer nodes, avoids the need to adaptively mesh, allows for easier design changes, deals with infinite domains and calculates gradients more accurately. Multiple commercial software packages have been created that use this method and include charged particles, allowing for potential and flux solutions in otherwise untenable complex geometries [50, 51, 52].

The boundary element method rapidly solves for the force on the surface without meshing the volume of the domain and handles arbitrary geometries by only discretizing points along that boundary. For example, consider Poisson's equation:

$$
\Delta U(\bar{x})=-\frac{\breve{\rho}}{\varepsilon_{0}},\left.U\right|_{\partial \Omega}=\text { for }\left.\nabla U\right|_{\partial \Omega} \cdot \vec{n}=g, \bar{x} \in \Omega
$$

where $\breve{\rho}$ is the charge density, $f$ is the Dirichlet fixed-potential $(U=$ const $)$ and $g$ is the Neumann fixed-flux boundary condition $\left(\frac{d U}{d \bar{x}}=\right.$ const $)$. A solution to Poisson's equation can be formed using a Green's function approach.

\section{Green's function}

Let $\mathrm{G}\left(\bar{x}, \bar{x}_{0}\right)$ be the free space Green's function for the Laplace operator, which is the solution to

$$
\Delta G=\delta\left(\bar{x}-\bar{x}_{0}\right)
$$


where $\bar{x}_{i}=\left(r_{i}, z_{i}\right)$ is a boundary point and $\bar{x}_{0}$ is a point on the interior of the domain. The Green's function in 3D coordinates is Eq. (11),

$$
G\left(\bar{x}, \bar{x}_{0}\right)=\frac{1}{4 \pi} \frac{1}{\left|\bar{x}-\bar{x}_{0}\right|}=\frac{1}{4 \pi} \frac{1}{\sqrt{\left(x-x_{0}\right)^{2}+\left(y-y_{0}\right)^{2}+\left(z-z_{0}\right)^{2}}}
$$

while in cylindrical coordinates it becomes Eq. (12).

$$
G\left(\bar{x}, \bar{x}_{0}\right)=\frac{1}{4 \pi} \frac{1}{\sqrt{r^{2}+r_{0}^{2}-2 r r_{0} \cos \left(\theta-\theta_{0}\right)+\left(z-z_{0}\right)^{2}}}
$$

Additionally, define two difference variables $L$ and $m$. In a $2 \mathrm{~d}$ axisymmetric model, these differences plus manipulation produce the equivalent Green's function, Eq. (15).

$$
\begin{gathered}
L=\left(r+r_{0}\right)^{2}+\left(z-z_{0}\right)^{2} \\
m=\frac{4 r r_{0}}{L} \\
G\left(\bar{x}, \bar{x}_{0}\right)=\frac{1}{\pi \sqrt{L}} \int_{0}^{\frac{\pi}{2}} \frac{d \theta}{\sqrt{1-m \sin ^{2} \theta}}=\frac{K(m)}{\pi \sqrt{L}}
\end{gathered}
$$

The $K(m)$ function is termed a complete elliptic integral of the first kind [53] and can be rapidly numerically computed using a method of arithmetic-geometric means [54, 55]. , As described in the following section, Green's functions are useful partly because they can be used to calculate the electrostatic potential at arbitrary points within the domain.

\section{Green's $2^{\text {nd }}$ identity}

Green's $2^{\text {nd }}$ identity mathematically relates an arbitrary surface contour to its boundary flux.

$$
\iint_{A}(D \Delta E-E \Delta D) d A=\oint(D \nabla E-E \nabla D) \cdot \vec{n} d S
$$

In Eq. (16), replace $D$ by the Greens function $G$ and $E$ by the potential $U$, giving Eq. (17) where $\partial_{\vec{n}} \xi=(\nabla \xi) \cdot \vec{n}$ is the dot product of the gradient and the normal vector.

$$
\iint_{A} G \frac{\breve{\rho}(\bar{x})}{\varepsilon_{0}} d A-U\left(\bar{x}_{0}\right)=\oint\left[G\left(\partial_{\vec{n}} U\right)-U\left(\partial_{\vec{n}} G\right)\right] \cdot \vec{n} d S
$$

Since there is no internal charge in a perfect conductor, the interior charge density $\breve{\rho}\left(\bar{x}_{0}\right)=0$ and the potential at any point outside the shape can be calculated solely from the given potential and potential gradient boundary conditions. Taking the limits of the single and double layered potential gives the boundary element formulation from inside the domain [56].

$$
\frac{1}{2} U\left(\bar{x}_{0}\right)=\oint_{\partial \Omega} U(\bar{x}) \partial_{\vec{n}} G\left(\bar{x}, \bar{x}_{0}\right) \mathrm{d} S-\oint_{\partial \Omega} \partial_{\vec{n}} U(\bar{x}) G\left(\bar{x}, \bar{x}_{0}\right) \mathrm{d} S
$$

The surface integral $\partial \Omega$ consists of a mixture of $U(\bar{x})$ along Dirichlet $\left(\partial \Omega_{D}\right)$ and $\partial_{\vec{n}} U(\bar{x})$ along Neumann $\left(\partial \Omega_{N}\right)$ boundary conditions, where the total surface is the sum of these two types, or $\partial \Omega=\partial \Omega_{D}+\partial \Omega_{N}$. Note that the flux on Dirichlet and the potential on Neumann boundaries are unknown and need to be determined for a complete solution. Also note that since this is a surface integral, the order of the points on each surface is important; their normals must point inward and the surface transversed in a counterclockwise direction. 


\section{Combined matrix}

If $\bar{x}$ is on the boundary, then Eq. (18) can be used to calculate the potential from these mixed boundaries at an interior point $\bar{x}_{0}$. Equation (19) is the full numerical discretization for determining this potential solely from the arbitraryshaped boundaries for the $N_{D}$ fixed potential and $N_{N}$ fixed flux panels.

$$
\begin{aligned}
\frac{1}{2} U\left(\bar{x}_{0}\right)= & \sum_{i=1}^{N_{D}} U\left(\bar{x}_{i}\right) \oint_{\partial \Omega_{D, i}} \partial_{n} G\left(\bar{x}, \bar{x}_{0}\right) \mathrm{d} S+\oint_{\partial \Omega_{D}} U(\bar{x}) \partial{ }_{\vec{n}} G\left(\bar{x}, \bar{x}_{0}\right) \mathrm{d} S \\
& -\sum_{i=1}^{N_{N}} \partial_{\vec{n}} U\left(\bar{x}_{i}\right) \oint_{\partial \Omega_{N, i}} G\left(\bar{x}, \bar{x}_{0}\right) \mathrm{d} S-\oint_{\partial \Omega_{N}} \partial_{\vec{n}} U(\bar{x}) G\left(\bar{x}, \bar{x}_{0}\right) \mathrm{d} S
\end{aligned}
$$

An analogous expression, but slightly different discretization can be achieved if the $\partial_{\vec{n}} U\left(\bar{x}_{i}\right)$ and $U\left(\bar{x}_{i}\right)$ terms in Eq. (19) are replaced with unknowns $\varphi_{i}$ and $\bar{\varpi}_{i}$. Set $\varphi_{i}$ to zero for fixed potential panels and $\bar{\omega}_{i}$ to zero for fixed potential flux panels [55]. With these substitutions, the simpler computational setup to determine the panel strengths for the panels can be written as Eq. (20).

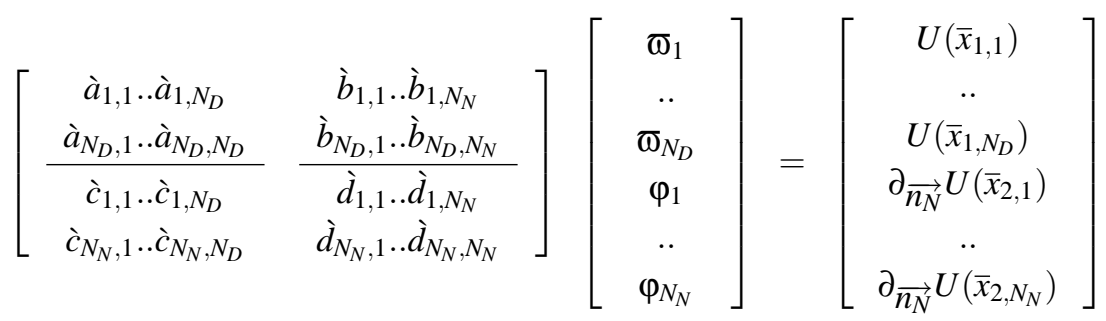

The shorthand values in the overall matrix are then given by Eq. (21). All the $\grave{a}_{i, j} . . \grave{d}_{i, j}$ terms are Green's functions or their derivatives and can be directly computed. Similarly, the $U_{D}\left(\bar{x}_{i, j}\right)$ and $\partial_{\overrightarrow{n_{N}}} U\left(\bar{x}_{i, j}\right)$ are given boundary condition values. The unknowns in the system are the relative strengths of these panels, the $\varpi$ and $\varphi$ vectors. Through a variety of methods such as full Gaussian inversion, iterative solving or matrix reduction, these unknowns can be mathematically determined.

$$
\left\{\begin{array}{l}
\grave{a}_{i, j}=\int_{\partial \Omega_{D_{i}}} \partial_{\overrightarrow{n_{D}}} G\left(\bar{x}, \bar{x}_{j}\right) \mathrm{d} S+\left\{\frac{1}{2} \in i=j\right\} \\
\grave{b}_{i, j}=\int_{\partial \Omega_{N_{i}}} G\left(\bar{x}, \bar{x}_{j}\right) \mathrm{d} S \\
\grave{c}_{i, j}=\int_{\partial \Omega_{D_{i}}} \partial_{\overrightarrow{n_{D}}} \partial_{\overrightarrow{n_{N}}} G\left(\bar{x}, \bar{x}_{j}\right) \mathrm{d} S \\
\grave{d}_{i, j}=-\int_{\partial \Omega_{N_{i}}} \partial_{\overrightarrow{n_{N}}} G\left(\bar{x}, \bar{x}_{j}\right) \mathrm{d} S+\left\{\frac{1}{2} \in i=j\right\}
\end{array}\right.
$$

Once the previously unknown values of $\left\{\Phi_{1} . . \Phi_{N_{D}}\right\}$ and $\left\{\varphi_{1} . . \varphi_{N_{N}}\right\}$ have been calculated, they can then be input back into the original discretization and used to calculate the potential anywhere in the domain through Eq. (22).

$$
U(\bar{x})=\sum_{j=1}^{N_{D}} \varpi_{j} \int_{\partial \Omega_{D}} \frac{\partial G\left(\bar{x}, \bar{x}_{j}\right)}{\partial n_{j}} d S+\sum_{j=1}^{N_{N}} \varphi_{j} \int_{\partial \Omega_{N}} G\left(\bar{x}, \bar{x}_{j}\right) d S
$$

Via this formula, the electric field as the gradient of the potential is available at any point throughout the interior of the domain.

\section{Panel force values}

To calculate the electrostatic force $q E$ on the surface listed in Eq. (3), consider the position and gradient among the droplet's electrons. Gauss' Law says that the total flux of the electric field through an element $d A$ is given by the sum of the electric field's surface normal; this is shown in Eq. (23).

$$
U L=\int E d A=\oint \vec{E} \cdot \vec{n} d S
$$

Drawing a box around each computational panel, the earlier assumption of the fluid being a perfect conductor means that all electric flux is normal to the surface so $\vec{E} \equiv \vec{E}_{n}$ and therefore there is not a tangential electric field. The entire contribution to the potential must come from the product of the normal electric field and the panel length, $L$. The electric field of a single body is determined by its total charge $q$, so the net electrostatic potential on a panel of length $L$ is $U=L E_{n}=\frac{q}{\varepsilon_{0}}$. Solving for the charge: 


$$
q=\varepsilon_{0} L E_{n}
$$

As a result, the total force experienced on the panel is given by Eq. (25).

$$
F=q E_{n}=\varepsilon_{0} L E_{n}^{2}
$$

This calculation method for the electrical force holds for any complex geometry, including when multiple shapes are nested inside one another. Anytime a nonzero electric field exists and is known, the number of electrons on the surface of a perfect conductor can be calculated.

\section{Time of flight algorithm}

With the ability to simulate forces on a detached droplet, there exists a desire to compare modeled results to published experimental droplet behavior. Literature searches refer to characteristic field emission-caused droplet detachment from colloid and FEEP needle time of flight (TOF) tests [57, 58]. These studies commonly have a needle, an extraction electrode and then a distant current collector plate.

A TOF measurement is a time-dependent spectrum of a current signal $I(t)$ following its instantaneous interruption. An experimental setup used by Gamero-Castaño is shown in Fig. (4) [59]. The emitter is operated in a steadystate mode and then is abruptly turned off. The stream of droplets moves past the extractor and towards the distant collector, $C_{T O F}$; these droplets are accelerated to different final velocities depending on their relative mass to charge ratio (MTCR). Droplet time of flight is a way to determine values for many characteristics of charged electrospray beams [60]. The spectrometers utilize the principle that particles of different masses with the same energy $E_{\text {part }}$ travel with different velocities inversely proportional to the square root of the mass [61].

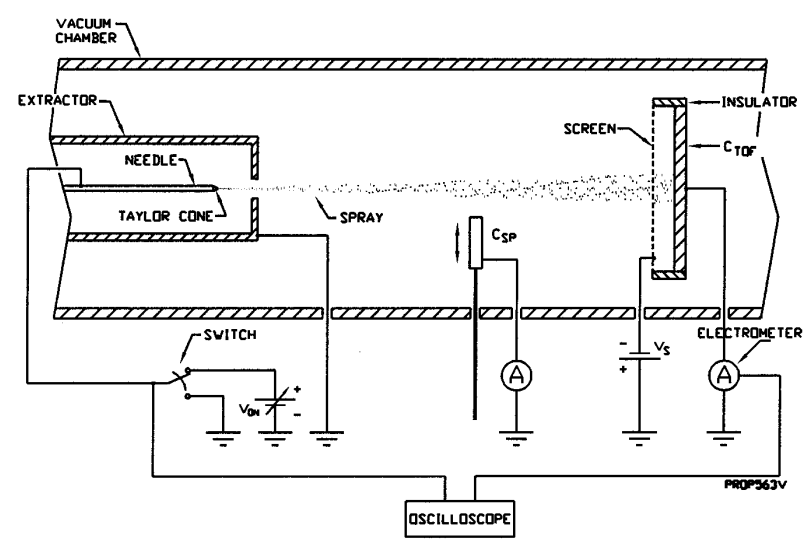

Figure 4. Experimental TOF setup used by Gamero-Castaño [59]

$$
V=\sqrt{\frac{2 E_{\text {part }}}{m}}
$$

The time-of-flight time $t_{T O F}$ of the particle over a fixed distance $d_{T O F}$ is therefore directly proportional to the square root of the mass. If this particle is a droplet with charge $q$ which has traveled through a potential $\Delta U$, the time of flight and mass per charge are given in Eq. (27).

$$
\begin{aligned}
t_{T O F} & =d_{T O F} \sqrt{\frac{m}{2 E}} \\
\frac{m}{q} & =2 \Delta U\left(\frac{t_{T O F}}{d_{T O F}}\right)^{2}
\end{aligned}
$$

The analysis of the current signal over time yields the specific charge distribution function of the droplets. By integrating the current, the thrust, mass flux, specific impulse and propulsive efficiency can be computed, as in Eq. (28). 


$$
\left\{\begin{aligned}
T & =\int_{0}^{\infty} \frac{2 U(t)}{d_{T F F}}[t I(t)] d t \\
\dot{m} & =\int_{0}^{\infty} \frac{2 U(t)}{d_{T O F}^{2}}\left[t^{2} I(t)\right] d t \\
I_{s p} & =\frac{T}{\dot{m} g} \\
\eta_{\text {prop }} & =\frac{T^{2}}{2 \dot{m} U I}
\end{aligned}\right.
$$

Using these equations, a simulated time of flight curve can be generated from a chosen starting design. Define the vector $b$ such that $b_{i}$ denotes the number of droplets produced at the needle in time $\tau$ with an acceleration $\alpha$ in the interval $\left[a_{i-1}, a_{i}\right)$ for $1 \leq i \leq N$ acceleration intervals. Since $\mathrm{F}=\mathrm{ma}=\mathrm{qE}, a_{i}=$ const $\times \frac{1}{M T C R_{i}} \times E_{i}$. Let $a_{1}$ be the minimum and $a_{N}$ be the maximum acceleration. Since all droplets are positively charged, $a_{1}, a_{N}>0$. Next, define the flux distribution function $\Xi(\alpha)$ to represent the number of particles produced per unit time with an acceleration in the interval $[\alpha, d \alpha)$.

$$
\Xi(\alpha) d \alpha=\frac{1}{t_{T O F}} \sum_{i=1}^{N} H\left(\alpha-a_{i-1}\right) H\left(a_{i}-\alpha\right) \frac{b_{i}}{a_{i}-a_{i-1}} d \alpha
$$

$\mathrm{H}(\mathrm{x})$ is the bimodal Heaviside step function as defined in Eq. (30).

$$
H(x-c) H(d-x)=\left\{\begin{array}{l}
1 \text { if } x \in[c, d) \\
0 \text { otherwise }
\end{array}\right.
$$

Since the thruster is operated in a steady state before emission cutoff, $\Xi(\alpha, t)=\Xi(\alpha)$ and the initial velocity of the droplets leaving the needle is effectively zero. The number of droplets $f(x, \alpha)$ in the spatial location $[\mathrm{x}, \mathrm{x}+\mathrm{dx})$ with acceleration $\alpha \in[\alpha, \alpha+d \alpha)$ is then given by Eq. (31), which multiplies the number of particles produced per time by the renormed velocity. The droplet's acceleration as a function of mass to charge is $\alpha=\frac{8.453 \times 10^{14}}{M T C R}$. Larger blobs with relatively fewer charges accelerate slower, so the minimum acceleration comes from the largest mass to charge ratio.

$$
\begin{aligned}
f(x, \alpha) & =\iint[\Xi(\alpha) d \alpha][t(x, \alpha) d x] \\
& =\frac{1}{t_{T O F}} \int_{\alpha_{\min }}^{\alpha_{\max }} \int_{0}^{x_{\text {needle }}} \sum_{i=1}^{N} H\left(\alpha-a_{i-1}\right) H\left(a_{i}-\alpha\right) \frac{b_{i}}{a_{i}-a_{i-1}} \frac{1}{\sqrt{2 x \alpha}} d \alpha d x
\end{aligned}
$$

Simulated times of flight for a needle FEEP are shown in the results, Sec. (IV-C).

\section{E. Minimum MTCR}

Efforts to determine the minimum MTCR of a charged droplet emitted from a surface began over one hundred years ago; Lord Rayleigh showed that for spherical droplet of radius $r_{\text {drop }}$, surface tension $\sigma$ and charge q, it does not break apart as long as the fissility $\chi$ does not exceed unity [62].

$$
\chi \leq \frac{q^{2}}{64 \pi^{2} \varepsilon_{0} \sigma r_{\text {drop }}^{3}}
$$

Above that point, the repulsive forces among the electrons outweigh the attractive force of surface tension. The droplet is then unstable and breaks apart, releasing some mass and the excess electrons into smaller satellite droplets. This event is known as Rayleigh discharge or Coulomb fission [63]. Thompson and Engel rewrote Rayleigh's expression using atomic mass $m$ and density $\rho$ [64]. For indium, droplet critical fissility is more easily calculated with this version. The rightmost term reports the breakup point in $\left[\frac{\mathrm{kg}}{\mathrm{C}}\right]$, while the factor $1.148 \times 10^{-6}$ converts it to the number of indium atoms per free electron. Recall that a low MTCR equates to a relatively greater charged droplet.

$$
1.148 \times 10^{-6} M T C R=\frac{m}{q} \frac{[k g]}{[C]} \geq \sqrt{\frac{\rho^{2} r_{d r o p}^{3}}{36 \varepsilon_{0} \sigma}}
$$

The minimum critical MTCR for a $1 \mu \mathrm{m}$ diameter droplet is approximately 160,000 indium atoms per free electron. Experimental results for indium droplets average approximately 100,000 atoms/electron [65]. Modeled droplet breakup is shown in Sec. (IV-D). 


\section{Results}

Using the methods and algorithms in Sec. (III), droplet detachment can be modeled and droplet dynamics can be analyzed. The mass to charge ratio and droplet diameter probability density functions and droplet time of flight, Rayleigh charge limit and velocity are presented as examples of these dynamics. The base computational case matches the experimental setup at ARCS. As shown initially in Fig. (2), it has a 2D axisymmetric needle field emitter with indium propellant and ring electrodes surrounding the tip. At $440 \mathrm{~K}$, indium has a viscosity of $1.17 \times 10^{-3} \frac{\mathrm{N} \cdot s}{\mathrm{~m}^{2}}$ and a surface tension of $0.552 \frac{\mathrm{N}}{\mathrm{m}}$. The computational domain is shown as Fig. (5). Along the right and top edges is imposed a zero potential flux condition, $\frac{d U_{n}(\bar{x})}{d \bar{x}}=0$. The potential on the electrode is chosen so that the needle tip experiences an electric field of $1 \times 10^{9} \frac{\mathrm{V}}{\mathrm{m}}$. Simulation run time for a pre-adaptively refined mesh on a $10 \mu \mathrm{m} \times 30 \mu \mathrm{m}$ grid with 800 boundary panels is a half day on a $2.5 \mathrm{GHz}$ Linux machine. An example of evolving droplet dynamics is given in Fig. (6). Previous results showing how the shape varies with viscosity, surface tension, etc. were presented in [66].

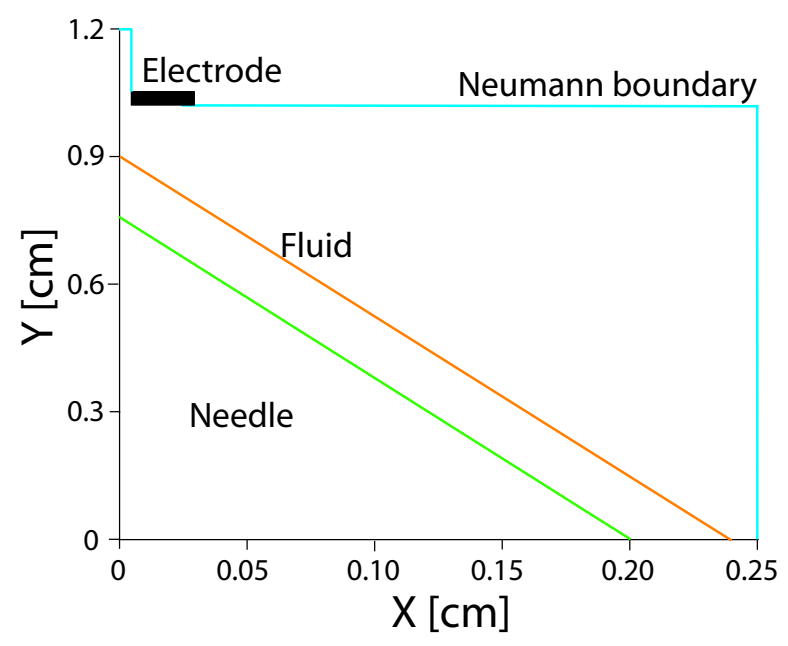

Figure 5. Computational boundary

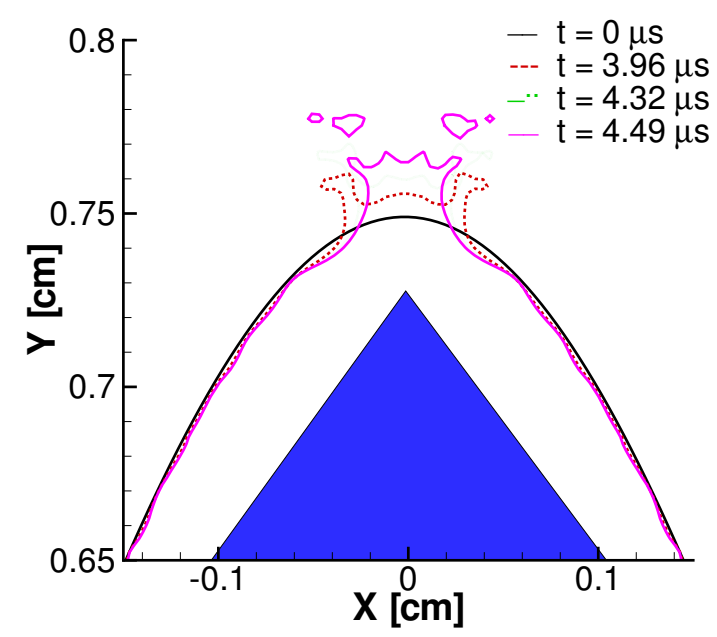

Figure 6. Sample droplet detachment 


\section{A. Mass to charge ratio}

The probability density function for 300 simulated detached droplets give the number of indium atoms per electron MTCR as shown in Fig. (7). The largest concentration with $10 \%$ of the total drops gives a relative MTCR around 3,800 , with a lower secondary peak around 200 atoms/electron. The individual mass and charge PDFs that comprise the MTCR figure are displayed in Fig. (8). There are strong probability peaks for the droplets at $3.3 \times 10^{-10} \mathrm{~kg}$ and $5 \times 10^{-8} \mathrm{C}$ with secondary concentrations at $2.8 \times 10^{-10} \mathrm{~kg}$ and $1.4 \times 10^{-7} \mathrm{C}$. The mass distribution fits especially well to a bigaussian distribution, with an RMS error of less than 3\%. A more detailed explaination for the location of the mass concentration is presented in the following subsection.

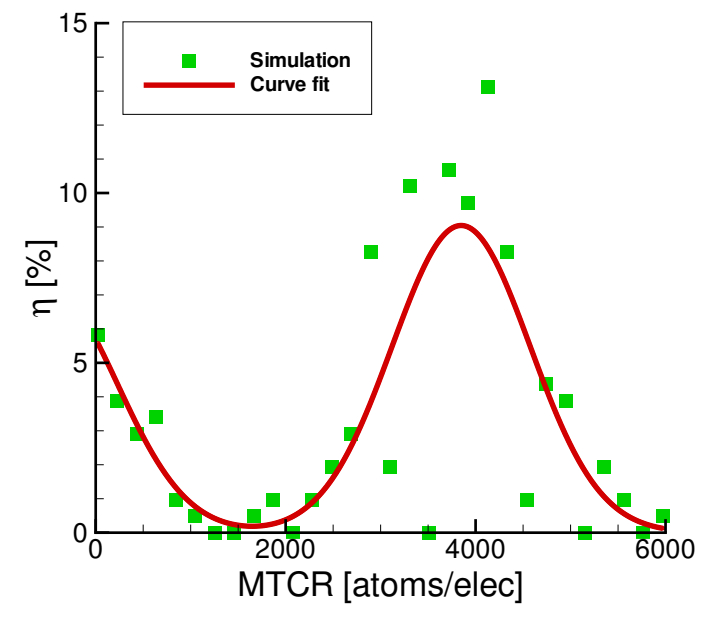

Figure 7. Droplet mass to charge probability density function

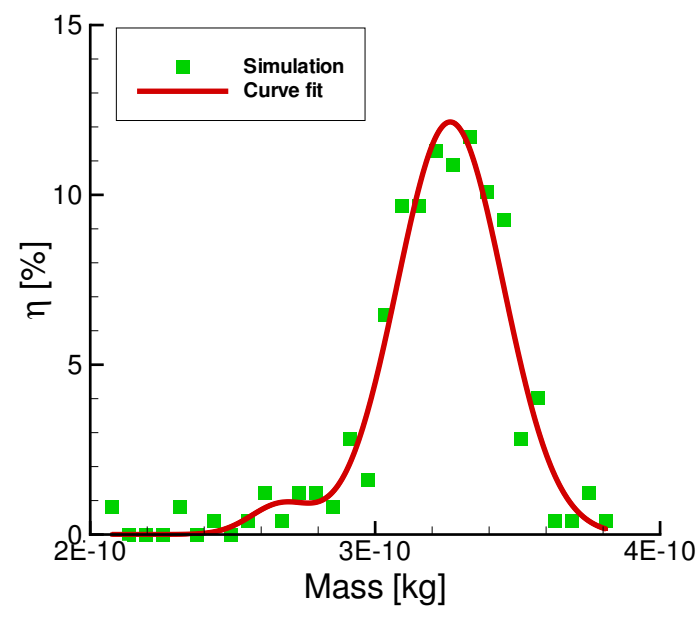

a)

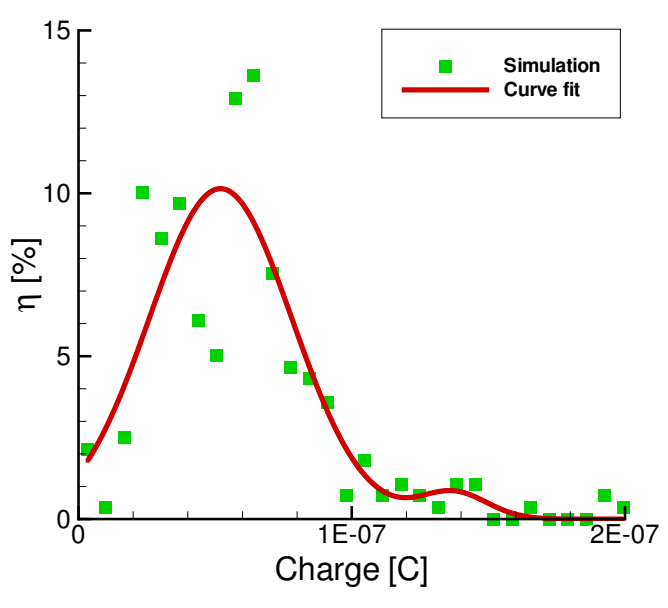

Figure 8. Droplet a) mass and b) charge probability density functions

The final velocity of a particle starting from rest in a constant electric field $E$ traveling over a fixed distance $x$ is $v^{2}=2 a \Delta x$.

$$
v=\sqrt{2 \frac{q}{m} E \Delta x}
$$


For the modal mass to charge of Fig. (7), Eq. (34) gives a droplet velocity of $1,600 \frac{\mathrm{m}}{\mathrm{s}}$; this is solidly within the measured velocity range for droplet flows in colloid thrusters [67].

\section{B. Droplet diameter}

In addition to the droplet diameter, the simulation resolves charge and mass. The probability density function for the the droplet diameter is shown in Fig. (9). The PDFs are presented for both modeled runs and a combined AFM/SEM normalized histogram from ARCS. The modeled diameter is determined by taking the area of the droplet and calculating the diameter of the equivalent circle. The experimental histogram mode of a $0.1 \mu \mathrm{m}$ diameter was arrived at similarly, by converting the volume of a droplet on a plate to that of a sphere with a comparable diameter. The height and relative peak width (st.dev./diameter) of the PDFs are similar, but unfortunately Seiborsdorf's setup details are unknown so only a qualitative comparison is possible [65].
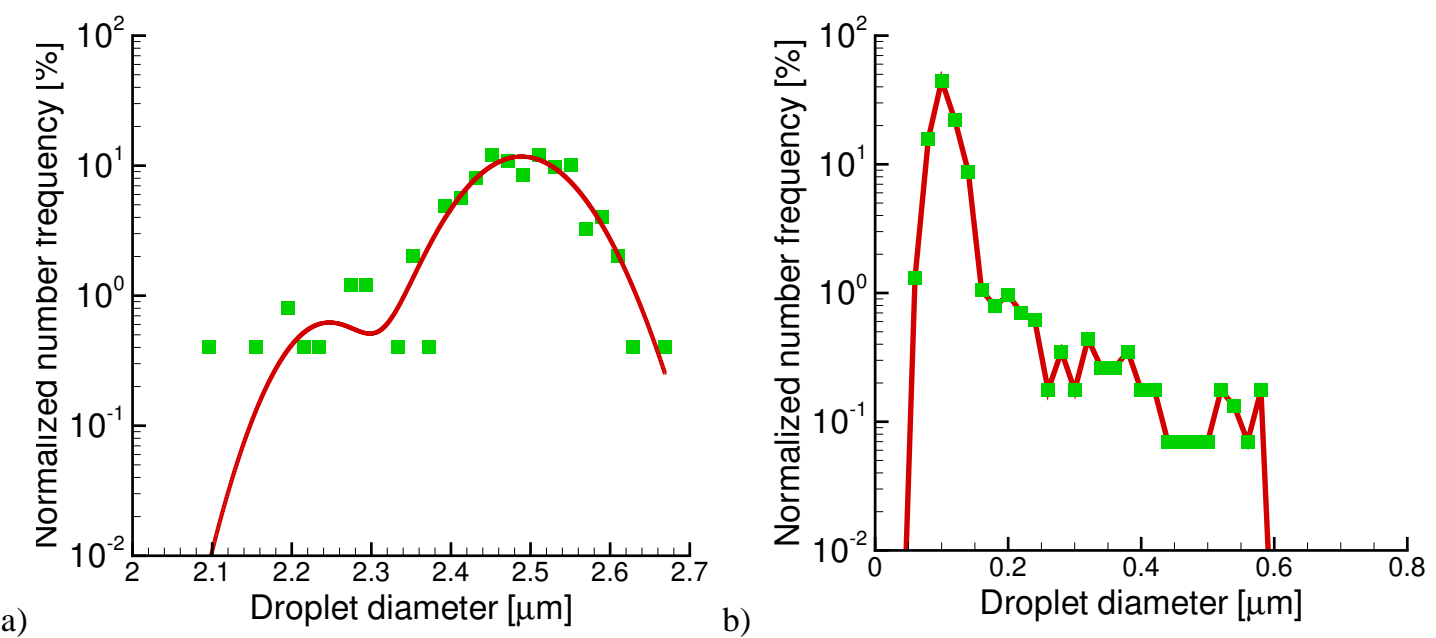

Figure 9. Droplet diameter probability density function for a a) modeled and b) ARCS experimental needle FEEP [65]

The modeled PDF most probable droplet diameter of $2.5 \mu \mathrm{m}$ can be explained through a look at forming microjets on top of the Taylor cone. Space charge effects near an infinitely fine cone point prevent field evaporation; this can be avoided by allowing for a small jet on top of the underlying Taylor shape. The size of these small extended jets vary depending on mass flow rates and tip radii of curvature. These protrusions have been observed at approximately 100 $\mathrm{nm}$ long and $30 \mathrm{~nm}$ in diameter[68, 69, 70]. The width of the jet $d_{\text {jet }}$ scales as Eq. $(35)[71,72]$.

$$
d_{\text {jet }}[m]=0.02 \sqrt{\frac{m_{\text {ion }} I}{\pi \rho q v}}
$$

At the point of jet initiation, field emission occurs at the tip and the surrounding fluid surface. An $800 \mu A$ current was simulated in the environment of Fig. (4). Together with the mass and charge PDF modes and $1 \frac{\mathrm{m}}{\mathrm{s}}$ fluid flow, the microjet diameter $d_{j e t}=0.3 \mu \mathrm{m}$. Using this value as an effective equivalent inner capillary diameter, the jet flow velocity assumption of Scheele can be used [73] as Eq. (36).

$$
v_{j e t}=1.73 \sqrt{\frac{\sigma}{\rho d_{j e t}}\left(1-\frac{d_{j e t}}{d_{\text {drop }}}\right)}
$$

Solving for the expected droplet diameter $d_{d r o p}$ gives a projected width of detached points as $2.3 \mu \mathrm{m}$, in accord with modeled results.

\section{Time of flight}

The two main droplet populations of Fig. (7) result in a bimodal acceleration peak, as shown in Fig. (10). A constant acceleration from the fixed potential difference produces increasing droplet velocities as they travel towards the elec- 
trode. Therefore, the density of droplets further from the needle tip in a given time interval decreases, as described in Sec (III-D). The largest droplet concentration occurs at the acceleration corresponding to the maximum droplet MTCR probability.

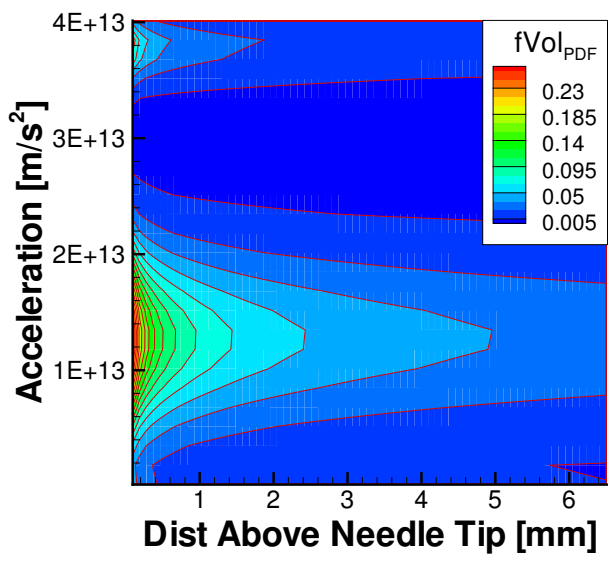

Figure 10. Probabilistic density contour of droplet axial position versus acceleration

Using Eq. (31) with the acceleration distribution of Fig. (10), a simulated time of flight current profile for a needle FEEP positioned in the experimental apparatus of Fig. (4) can be determined. Figure (11) shows the current recorded on the collector plate over time for a hypothetical $800 \mu \mathrm{A}$ multi-emitter configuration and that of an experimental colloid TBP1 thruster. The acceleration experienced by the indium droplets due to their being a perfect conductor, more highly charged and the electrode's higher potential difference results in the significant reduction in time of flight predicted in comparison to measured colloid data. However, the shape of the simulated curve matches well with the minimum droplet flow regime of $28 \mathrm{nA}$.

a)

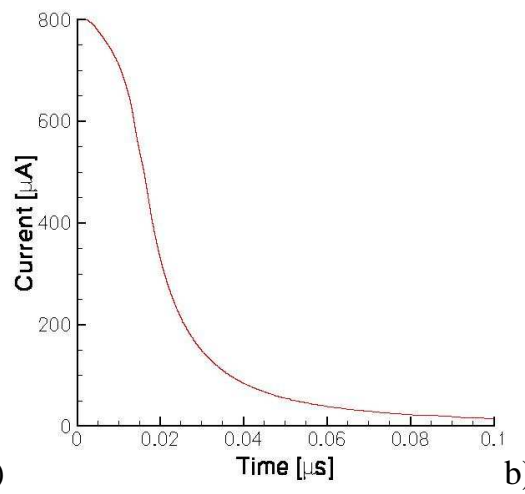

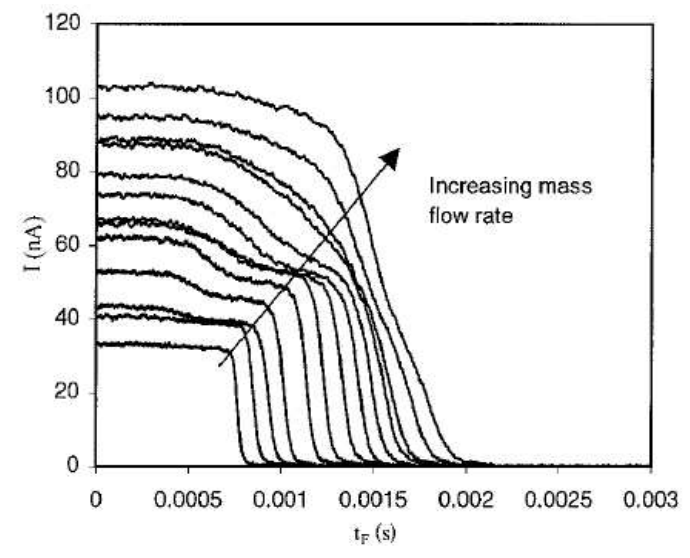

Figure 11. Droplet time of flight current profile for a) simulated indium FEEP and b) experimental TBP1 colloid emitter [74]

\section{Rayleigh limit}

As the droplets move upward towards the electrode, they often fission into smaller droplets. When this occurs, the mass to charge ratio increases because the relative charge decreases on the remaining droplet. The plot of a single droplet's relative charge versus distance along the axis is shown in Fig. (12). The original needle tip is at $0.75 \mathrm{~cm}$. The droplet breaks into multiple new points at a height of $0.762 \mathrm{~cm}$ and $0.768 \mathrm{~cm}$. 


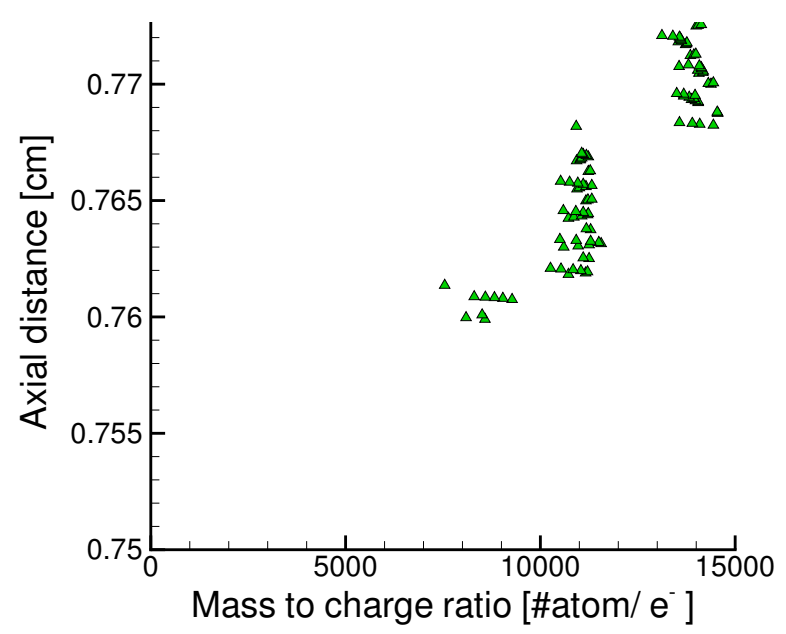

Figure 12. Droplet mass to charge history of one droplet moving upward along the axis

\section{Conclusion}

The coupling of the boundary element and level set methods allows for the simulation of liquid indium forming droplets off a needle FEEP tip. The boundary element method (BEM) provides a rapid calculation of the external potentials and normal electric fields, thereby driving the propellant evolution. Due to the surface-only character of the BEM algorithm, an arbitrary, complex and time-variant geometry is able to be simulated. An electrostatic potential difference is the main external force driving the combined-level- set-volume-of-fluid (CLSVOF) $\phi$ updating. The combination of the two methods provides a second order spatially accurate simulation of a 2D axisymmetric electrode inducing droplet formation. Droplet diameters and charges were analyzed after breakoff. Initial charge/mass ratio, acceleration and speed were computed for droplets detaching from a needle FEEP and a single droplet's fissioning history was shown.

\section{Acknowledgments}

The first author thanks the NASA Jet Propulsion Laboratory for funding through the NASA Graduate Student Research Program.

\section{References}

1 Paine, M. and Gabriel, S., "A micro-fabricated colloidal thruster array," No. AIAA-2001-3329 in 37th AIAA Joint Propulsion Conference, Salt Lake City, Utah, 8 July 2001.

2 Schottky, W., “Concerning cold and hot electron discharge," Zeitschrift fur Physik, Vol. 14, 1923, pp. 63.

3 Iribarne, J. V. and Thomson, B. A., "Evaporation of small ions from charged droplets," J. Chemical Physics, Vol. 64, No. 6, 1976, pp. 2287-2294.

4 Gamero-Castaño, M. and Fernández de la Mora, J., "Direct measurement of ion evaporation kinetics from electrified liquid surfaces," J. Chemical Physics, Vol. 113, No. 2, 8 July 2000, pp. 815-832.

5 Tsong, T. T. and Müller, E. W., "Measurement of energy distribution in field ionization," J. Chemical Physics, Vol. 41, No. 11, 1964, pp. 3279.

6 Hong, Y. C. and Uhm, H. S., "Production of carbon nanotubes by microwave plasma torch at atmospheric pressure," Physics of Plasmas, Vol. 12, No. 5, May 2005, Art. No. 053504. 
7 Bouchard, R. J., Cheng, L. T., Roach, D. H., Moffett, P. J., and Hang, K. W., "Screen printable dielectric for field emission displays,” J. American Ceramic Society, Vol. 88, No. 6, June 2005, pp. 1465-1467.

8 Busta, H., Tolt, Z., Montgomery, J., and Feinerman, A., "Field emission from teepee-shaped carbon nanotube bundles," J. Vacuum Science and Technology B, Vol. 23, No. 2, March 2005, pp. 676-679.

9 Sossi, V., Buckley, K. R., Piccioni, P., Rahmim, A., Camborde, M. L., Strome, E., Lapi, S., and Ruth, T. J., "Printed sources for positron emission tomography (PET)," IEEE Transactions on Nuclear Science, Vol. 52, No. 1, Feb. 2005, pp. 114-118.

10 Chung, M., Miskovsky, N. M., Cutler, P. H., Kumar, N., and Patel, V., "Theoretical analysis of a field emission enhanced semiconductor thermoelectric cooler," Solid-State Electronics, Vol. 47, No. 10, Oct. 2003, pp. 17451751.

11 Yahiku, A. Y., Mahoney, J. F., Daley, H. L., Perel, J., and Sherman, A., "Experimental study of colloid annular thrusters," No. AIAA-70-1112 in 8th Electric Propulsion Conference, Stanford, California, Sept. 1970.

12 Daley, H. L., Mahoney, J. F., and Perel, J., “Colloid annular array thruster development,” No. AIAA-73-1077 in 10th Electric Propulsion Conference, Lake Tahoe, New York, Nov. 1973.

13 Lozano, P. and Martinez-Sánchez, M., "Experimental Measurements of Colloid Thruster Plumes in the Ion-Droplet Mixed Regime,” No. 2002-3814 in AIAA 38th Joint Propulsion Conference, Indianapolis, IN, July 2002, pp. 1-7.

14 Nicolini, D., Marcuccio, S., and Andrenucci, M., “3-D Plume Characterization of a FEEP Thruster," No. 20003269 in AIAA 36th Joint Propulsion Conference, Centrospazio, Huntsville, AL, July 2000, pp. 1-4.

15 Carretero, J. and Martinez-Sanchez, M., "Quasi-one-dimensional numerical simulation of a single-emitter colloidal jet," Vol. AIAA 2002-3812 of 38th Joint Propulsion Conference, Indianapolis, Indiana, 7-10 July 2002, pp. 1-7.

16 Tajmar, M., Steiger, W., and Genovese, A., "Indium FEEP Thruster Beam Diagnostics, Analysis and Simulation," No. 2001-3790 in JPC 37th Joint Propulsion Conference, Austrian Research Centre Seibersdorf, Salt Lake City, UT, July 2001.

17 Genovese, A., Tajmar, M., and Steiger, W., "Indium FEEP endurance test: preliminary results,” No. IEPC-01-289 in IEPC 27th International Electric Propulsion Conference, Pasadena, CA, October 2001.

18 Berti, E., Buonanno, A., and Will, C. M., "Estimating spinning binary parameters and testing alternative theories of gravity with LISA," Physical Review D, Vol. 71, No. 8, April 2005.

19 Karlsson, A., "Darwin mission,” 2006, http://darwin.esa.int/science-e/www/area/index.cfm?fareaid=28.

20 Kern, M. and et al., "Outlier detection algorithms and their performance in GOCE gravity field processing," $J$. Geodesy, Vol. 78, No. 9, April 2005, pp. 509-519.

21 Marirrodriga, C. G., "ESA takes another step towards the search for Earth-like planets," 2001, http://www.esa.int/esaCP/ASEKEVNW9SC_Expanding_0.html.

22 Tajmar, M., Genovese, A., Buldrini, N., and Steiger, W., "Miniturized Indium-FEEP Multiemitter Design and Performance," Vol. AIAA 2002-5718, AIAA NanoTech 2002, Houston, TX, 9 September 2002, pp. 1-7.

23 Tajmar, M., Marhold, K., and Kropatschek, S., “Three-Dimensional In-FEEP Plasmadiagnostics,” IEPC 28th International Electric Propulsion Conference, Toulouse, France, July 2003, pp. 1-10.

24 Farag, A. and Hassan, H., "Adaptive Segmentation of Multi-model 3D Data Using Robust Level Set Techniques," MICCAI LNCS, Vol. 3216, 2004, pp. 143-150.

25 Dean, J. A., editor, Lange's Handbook of Chemistry, McGraw-Hill, 15th ed., 1999, Table 6.4 - Heats of Fusion, Vaporization. Online at http://www.knovel.com/knovel2/Toc.jsp?BookID=47\&VerticalID=0.

26 Genovese, A., Steiger, W., and Tajmar, M., "Indium FEEP Microthruster: Experimental Characterization in the 1-100 $\mu$ N Range," Vol. AIAA 2001-3788 of 37th Joint Propulsion Conference, 8-11 July 2001, pp. 1-12. 
27 Eustathopoulos, N., Nicholas, M., and Drevet, B., Wettability at High Temperatures, Pergamon Materials Series, New York, USA, 1999.

28 Nave, C. R., "Modeling the radiative cooling of a hot sphere," Tech. rep., Department of Physics and Astronomy, Georgia State University, http://hyperphysics.phy-astr.gsu.edu/hbase/thermo/cootime.html\#c2, 2005.

29 Gibou, F., Fedkiw, R. P., Cheng, L. T., and Kang, M. J., "A second-order-accurate symmetric discretization of the Poisson equation on irregular domains," J. Computational Physics, Vol. 176, No. 1, 10 Feb. 2002, pp. $205-227$.

30 Osher, S. and Sethian, J., "Fronts propagating with curvature dependent speed: algorithms based on HamiltonJacobi formulations," J. Computational Physics, Vol. 79, 1988, pp. 12-49.

31 Osher, S. and Fedkiw, R., Level set methods and dynamics implicit surfaces, Vol. 153 of Applied Mathematical Sciences, Springer, 2003.

32 Sussman, M., Smereka, P., and Osher, S., "A level set approach for computing solutions to incompressible twophase flow," J. Computational Physics, Vol. 114, 1994, pp. 146-159.

33 Enright, D., Losasso, F., and Fedkiw, R., "A fast and accurate semi-Lagrangian particle level set method," Computers and Structures, Vol. 83, 2005, pp. 479-490.

34 Sussman, M., "A second order coupled level set and volume-of-fluid method for computing growth and collapse of vapor bubbles," J. Computational Physics, Vol. 187, 2003, pp. 110-136.

35 Arienti, M., Hung, P., Morano, E., and Shepherd, J., “A level set approach to Eulerian-Lagrangian coupling,” J. Computational Physics, Vol. 185, 2003, pp. 213-251.

36 Yu, J. D., Sakai, S., and Sethian, J., "A coupled quadrilateral grid level set projection method applied to ink jet simulation,” J. Computational Physics, Vol. 206, No. 1, 10 June 2005, pp. 227-251.

37 Chung, E. T., Chan, T. F., and Tai, X. C., "Electrical impedance tomography using level set representation and total variational regularization,” J. Computational Physics, Vol. 205, No. 1, 1 May 2005, pp. 357-372.

38 Yue, W. S., Lin, C. L., and Patel, V. C., "Large eddy simulation of turbulent open-channel flow with free surface simulated by level set method," Physics of Fluids, Vol. 17, No. 2, Feb. 2005, pp. No. 025108.

39 Leow, A., Yu, C. L., Huang, S. C., Protas, H., Nicolson, R., Hayashi, K. M., Toga, A. W., and Thompson, P. M., "Brain structural mapping using a novel hybrid implicit/explicit framework based on the level-set method," Neuroimage, Vol. 24, No. 3, 1 Feb. 2005, pp. 910-927.

40 Ben Ayed, I., Mitiche, A., and Belhadj, Z., "Multiregion level-set partitioning of synthetic aperture radar images," IEEE Transactions on Pattern Analysis and Machine Intelligence, Vol. 27, No. 5, May 2005, pp. 793-800.

41 Huang, B., Li, H., and Huang, X., "A level set method for oil slick segmentation in SAR images," International J. of Remote Sensing, Vol. 26, No. 6, March 2005, pp. 1145-1156.

42 Alexandrov, O., "Level set - matlab," Tech. rep., 14 May 2006, http://en.wikipedia.org/wiki/User:Olegalexandrov.

43 Ohta, M., Haranaka, S., Yoshida, Y., and Sussman, M., "Three-dimensional numerical simulations of the motion of a gas bubble rising in viscous liquids," J. of Chemical Engineering of Japan, Vol. 37, No. 8, 2004, pp. 968-978.

44 Sussman, M., Fatemi, E., Smereka, P., and Osher, S., "An improved level set method for incompressible two-phase flows," Computers \& Fluids, Vol. 27, No. 5-6, June 1998, pp. 663-680.

45 Godunov, S., Zabrodin, A., and Prokopov, G., USSR Comput. Math. Math. Phys., Vol. 1, 1961, pp. 1187.

46 Sussman, M., "A parallelized, adaptive algorithm for multiphase flows in general geometries," Computers and Structures, Vol. 83, 2005, pp. 435-444.

47 Sussman, M. and Puckett, E., "A coupled level set and volume-of-fluid method for computing 3D and axisymmetric incompressible two-phase flows," J. Computational Physics, Vol. 162, 2000, pp. 301-337. 
49 Greenbaum, A., Greengard, L., and McFadden, G. B., "Laplace's equation and the Dirichlet-Neumann map in multiply connected domains," J. Computational Physics, Vol. 105, 1993, pp. 267-278.

${ }^{50}$ Ivanov, V., Krasnykh, A., Scheitrum, G., and Jensen, A., “An improved version of TOPAZ3D,” Vol. 5 of Particle Accelerator Conference, 12 May 2003, pp. 3315-3317.

51 Shapiro, A., "TOPAZ3D : A three-dimensional finite element heat transfer code," Tech. rep., Aug. 1985, UCID20484. TOPAZ3D DEC VAX 11 Vesion Tape Description NESC Note 87-64 (March 24, 1987).

52 Kirkup, S., BEM CD 2005: the boundary element method, boundary-element-method.com, 2005.

53 Abramowitz, M. and Stegun, I., Handbook of mathematical functions, Dover Publications, Inc., 1974.

54 Shure, L. and Bonnevier, B., “MATLAB function ellipke,” Tech. rep., The Mathworks, Inc., 1988.

55 Emhoff, J., Simulation of ion optics using particle-in-cell and treecode methods, Ph.D. thesis, Dept. Aerospace Engineering, University of Michigan, 1320 Beal Avenue, Ann Arbor, MI, May 2005.

56 Folland, G., Introduction to Partial Differential Equations, Princeton University Press, 1979.

57 Chiu, Y.-H., Austin, B. L., Dressler, R. A., Levandier, D., Murray, P. T., Lozano, P., and Martinez-Sánchez, M., "Mass spectrometric analysis of colloid thruster ion emission from selected propellants," J. Propulsion and Power, Vol. 21, No. 3, May 2005, pp. 416-423.

58 Gamero-Castaño, M. and Hruby, V., "Electric Measurements of Charged Sprays Emitted by Cone-jets," Journal Fluid Mechanics, Vol. 459, 2002, pp. 245-276.

59 Gamero-Castaño, M. and Hruby, V., "Electrospray as a source of nanoparticles for efficient colloid thrusters," $J$. Propulsion and Power, Vol. 17, No. 5, Oct. 2001, pp. 977-987.

${ }^{60}$ Hunter, R. E. and Wineland, S. H., "Charged colloid generation research," Space Electronics Symposium, Los Angeles, CA, 1965.

61 Victor, A., Zurbuchen, T., and Gallimore, A., "Development of the top hat electric propulsion plume analyzer (TOPAZ): mass analyzer design and preliminary calibration data," No. 2005-016 in 29th International Electric Propulsion Conference, AIAA, Princeton, NJ, Nov. 2005, pp. 1-11.

62 Rayleigh, L. and Strutt, J. W., Phil. Mag., Vol. 14, 1882, pp. 184-186.

${ }^{63}$ Kebarle, P., J. Mass Spectrometry, Vol. 35, 2000, pp. 804.

64 Thompson, S. and von Engel, A., "Field emission of metal ions and microparticles," J. Physics D, Vol. 15, 1982, pp. $925-931$.

${ }^{65}$ Fehringer, M., Rudenauer, F., and Steiger, W., “Ultra-Precise Indium Thruster, WP 2000: Droplet Emission,” Tech. Rep. ESTEC Contract No. 12376/97/NL/PA, Seiborsdorf, Austria, 2000.

66 VanderWyst, A., Christlieb, A., Sussman, M., and Boyd, I., "Boundary integral formulation of electric fields in level set simulations of charged droplets," No. 2005-4665 in 36th AIAA Plasmadynamics and Lasers Conference, AIAA, Toronto, Canada, 6 June 2005.

${ }^{67}$ Marcuccio, S., Genovese, A., and Andrenucci, M., "Experimental performance of field emission microthrusters," J. Propulsion and Power, Vol. 14, No. 5, 1998, pp. 774-781.

${ }^{68}$ Swanson, L. W., "Electron and ion emission from liquid metal surfaces," IEEE Transactions on Plasma Science, Vol. 19, No. 5, Oct. 1991, pp. 746-748.

69 Vladimirov, V. and Gorshkov, V., "Stability of conducting beams in ionic sources on liquid metals," Journal of Technical Physics, Vol. 57, No. 11, 1987, pp. 2155-2163. 
70 Kang, N. K. and Swanson, L. W., "Computer simulation of liquid-metal ion-source optics," Applied Physics, Vol. A30, No. 2, 1983, pp. 95-104.

71 Vladimirov, V., Badan, V., and Gorshkov, V., "Microdroplet emission and instabilities in liquid-metal ion sources," Surface Science, Vol. 266, 1992, pp. 185-190.

72 Vladimirov, V. V. and Gorshkov, V. N., J. Appl. Phys. A, Vol. 46, 1988, pp. 131.

73 Scheele, G. F. and Meister, B. J., "Drop prediction at low velocities in liquid-liquid systems," J. AIChE, Vol. 14, No. 1, 1968, pp. 9-15.

74 Romero-Sanz, I., Bocanegra, R., and Fernández de la Mora, J., "Source of heavy molecular ions based on Taylor cones of ionic liquids operating in the pure ion evaporation regime," J. Applied Physics, Vol. 94, No. 5, 1 Sept. 2003, pp. 3599-3605. 